\title{
Representing Grounding Line Dynamics in Numerical Ice Sheet Models: Recent Advances and Outlook
}

\author{
David Docquier • Laura Perichon • Frank Pattyn
}

Received: 18 February 2011/ Accepted: 16 May 2011

(C) Springer Science+Business Media B.V. 2011

\begin{abstract}
Recent satellite observations of the Antarctic and Greenland ice sheets show accelerated ice flow and associated ice sheet thinning along coastal outlet glaciers in contact with the ocean. Both processes are the result of grounding line retreat due to melting at the grounding line (the grounding line is the contact of the ice sheet with the ocean, where it starts to float and forms an ice shelf or ice tongue). Such rapid ice loss is not yet included in large-scale ice sheet models used for IPCC projections, as most of the complex processes are poorly understood. Here we report on the state-of-the art of grounding line migration in marine ice sheets and address different ways in which grounding line migration can be attributed and represented in ice sheet models. Using onedimensional ice flow models of the ice sheet/ice shelf system we carried out a number of sensitivity experiments with different spatial resolutions and stress approximations. These are verified with semi-analytical steady state solutions. Results show that, in large-scale finite-difference models, grounding line migration is dependent on the numerical treatment (e.g. staggered/non-staggered grid) and the level of physics involved (e.g. shallow-ice/ shallow-shelf approximation).
\end{abstract}

Keywords Marine ice sheet instability · Grounding line - Ice sheet modeling · West Antarctic ice sheet

D. Docquier $(\bowtie) \cdot$ L. Perichon $\cdot$ F. Pattyn

Laboratoire de Glaciologie, Université Libre de Bruxelles, CP160/03, Av. F.D. Roosevelt 50,

1050 Brussels, Belgium

e-mail: david.docquier@ulb.ac.be

L. Perichon

e-mail: 1pericho@ulb.ac.be

F. Pattyn

e-mail: fpattyn@ulb.ac.be 


\section{Introduction}

The West Antarctic Ice Sheet (WAIS) is currently losing ice at a considerable rate (Chen et al. 2009; Velicogna 2009; Rignot et al. 2008, 2011). Pine Island (PIG) and Thwaites Glaciers, situated in the Amundsen Sea Embayment, are the main contributors to this WAIS mass loss (Shepherd et al. 2001). PIG in particular has shown a nearly continuous acceleration (Joughin et al. 2003) and thinning (Wingham et al. 2009) during recent years. Recent evidence shows that this thinning is due to an inland grounding line migration from the 1970 s to present by $30 \mathrm{~km}$ (Jenkins et al. 2010).

Due to its marine configuration, i.e. its bedrock mostly lying below sea level, the stability of the WAIS has been a subject of much debate. Marine ice sheet stability is mostly controlled by the dynamics of the grounding line, i.e. the junction between the grounded ice sheet and the floating ice shelf. This junction marks the change from inland ice sheet flow, dominated by vertical shear and basal friction, toward ice shelf flow, dominated by longitudinal stresses, and which is quintessential in understanding grounding line dynamics. Weertman (1974) and Thomas and Bentley (1978) proposed that ice discharge through the grounding line should increase with ice thickness. Therefore, a marine ice sheet lying on an upward-sloping bed (toward the ocean), such as WAIS, is unstable. A slight retreat in grounding line position could therefore lead to an increase in ice thickness, hence increased ice discharge at the grounding line. Ice sheet thinning is then initiated and further retreat of the grounding line potentially occurs.

Hindmarsh $(1993,1996)$ argued that ice shelves should have a limited impact on ice sheet dynamics, and that grounding line migration is governed by grounded ice flux. He advocated the concept of neutral equilibrium, i.e. that a perturbation in grounding line position should not lead to unstable retreat or advance for a foredeepened or upwardsloping bed, nor to a return to the original grounding line position for a downward-sloping bed.

However, Schoof (2007a, b) re-confirmed the instability hypothesis formulated by Weertman (1974) and Thomas and Bentley (1978), on the basis of a boundary layer theory for the ice sheet/ice shelf transition. He demonstrated that (1) marine ice sheets do not exhibit neutral equilibrium, but have well-defined, discrete equilibrium profiles; (2) steady grounding lines cannot be stable on upward-sloping beds; (3) marine ice sheets with overdeepened beds can undergo hysteresis under variations in sea level, accumulation rate, basal slipperiness and ice viscosity. Robison et al. (2009) confirmed the stability of the grounding line on a downward bed slope comparing fluid-mechanical experiments and model results (with time-dependent evolution of the grounding line), while Durand et al. (2009a) demonstrated the instability of marine ice sheets on upsloping beds with a Full Stokes (Elmer/Ice) model.

The aim of this paper is to address the state-of-the-art of modeling the processes affecting marine ice sheet stability and grounding line migration. To this end, we give an overview of different numerical approaches and approximations used in ice sheet models. A description is given for a simple model that copes with grounding line migration in a parameterized way. Numerical experiments are carried out to investigate the aspects of grid resolution and the time-dependent behavior and a discussion is given on the appropriate use of approximations and numerical approaches in large-scale ice sheet models. 


\section{Grounding Line Models: An Overview}

\subsection{Numerical Approaches}

There are several numerical approaches in ice sheet models to simulate grounding line migration: fixed grid (FG), moving grid (MG) and adaptive techniques. They essentially differ in the way grounding lines are represented. The majority of models make use of the flotation criterion to separate grounded and floating ice:

$$
\rho_{i} h=\rho_{w}\left(z_{s l}-b\right),
$$

where $\rho_{i}$ and $\rho_{w}$ are ice and water densities, respectively, $h$ is the ice thickness, $z_{s l}$ is the sea level elevation, $b$ is the bedrock elevation. In FG models, the grounding line position is not defined explicitly but must fall between grid points where ice is grounded and floating. Large-scale ice sheet models (Huybrechts 1990; Ritz et al. 2001) use this strategy to simulate grounding line migration. Moving grid (MG) models allow the grounding line position to be followed continuously, i.e. the grounding line coincides exactly with a grid point (Hindmarsh 1996; Hindmarsh and Le Meur 2001).

Vieli and Payne (2005) showed that there is a strong dependency of FG models on numerics, that-when perturbed-FG models exhibit large changes in grounding line migration, and that these changes are irreversible. Conversely, for MG models, changes in grounding line position are generally small and reversible. However, the models based on shallow-ice approximation used by Vieli and Payne lack a second independent boundary condition that is needed to accurately represent grounding line migration (see below; Schoof 2007a; Durand et al. 2009a), and although MG models generally produce more consistent results, a major drawback remains the complexity to implement in a threedimensional ice sheet model (Vieli and Payne 2005).

Adaptive models are a trade-off between FG and MG models. Durand et al. (2009a) used the finite element code Elmer/Ice to couple the Stokes equations with the evolution of two free surfaces, i.e. the upper interface (air/ice) and the bottom interface (ice/bed or ice/ sea). They applied a mesh refinement around the grounding line. With this method, the total number of nodes is constant and only the horizontal distribution of the nodes is modified. Durand et al. (2009a) need a grid size below $100 \mathrm{~m}$ at the grounding line in order to achieve consistent results.

Goldberg et al. (2009) used adaptive refinement, i.e. cells are divided into smaller cells where extra resolution is required and groups of cells are coarsened into larger cells in regions where lower resolution suffices. The grounding line is represented either as lying at the boundary between entirely grounded and entirely floating cells or by interpolating the flotation condition between grid lines according to Pattyn et al. (2006) (cells can be partially grounded in the latter case). Goldberg et al. (2009) found that buttressing was not always sufficient to stabilize an ice sheet, but the collapse of the grounded portion was greatly delayed.

Katz and Worster (2010) presented a theory for grounding line dynamics in three spatial dimensions. Although they incorporated internal shear and membrane stresses, a Newtonian viscosity was used and basal sliding neglected. They also used the $2 \mathrm{D}$ ice shelf proposed by Robison et al. (2009), which is valid only when the grounding line position is independent of the transverse direction.

Finally, FG models can be adapted in such a way that sub-grid grounding line position and migration can be achieved through local interpolations and approximations. Pattyn 
et al. (2006) determined grounding line position at sub-grid resolution using the flotation criterion (1) and applied a basal friction function to mimic in a continuous way the transition zone between ice sheet and ice shelf. Gladstone et al. (2010) used several interpolation schemes in combination with locally increased resolution to suit the same purpose.

\subsection{Physical Approximations}

\subsubsection{Linear Momentum}

The flow of an ice body is described by the linear momentum balance equation:

$$
\rho_{i} \frac{d \mathbf{v}}{d t}=\nabla \cdot \tau+\rho_{i} \mathbf{g}
$$

where $\mathbf{v}$ is the velocity field, $\tau$ is the Cauchy stress tensor, and $\mathbf{g}$ is the gravitational acceleration. Neglecting acceleration terms and considering the gravitational acceleration only important in the vertical direction, we can write (2) in its component form (where $x$ is the flow direction, $y$ is the direction perpendicular to the flow and $z$ is the vertical direction, positively upward):

$$
\begin{gathered}
\frac{\partial \tau_{x x}}{\partial x}+\frac{\partial \tau_{x y}}{\partial y}+\frac{\partial \tau_{x z}}{\partial z}=0, \\
\frac{\partial \tau_{y x}}{\partial x}+\frac{\partial \tau_{y y}}{\partial y}+\frac{\partial \tau_{y z}}{\partial z}=0, \\
\frac{\partial \tau_{z x}}{\partial x}+\frac{\partial \tau_{z y}}{\partial y}+\frac{\partial \tau_{z z}}{\partial z}=\rho_{i} g .
\end{gathered}
$$

Full Stokes (FS) models (Durand et al. 2009a, b) solve the full system of linear momentum equations. Due to the considerable computational effort, approximations to these equations are often used, such as higher-order, shallow-shelf and shallow-ice approximations. They involve dropping terms from the momentum balance equations and simplifying the strain rate definitions.

Higher-order models (HOM) consider the hydrostatic approximation in the vertical direction by neglecting vertical resistive stresses, so that the linear momentum can be written (Herterich 1987; Blatter 1995; Pattyn 2003):

$$
\begin{gathered}
\frac{\partial \tau_{x x}}{\partial x}+\frac{\partial \tau_{x y}}{\partial y}+\frac{\partial \tau_{x z}}{\partial z}=0 \\
\frac{\partial \tau_{y x}}{\partial x}+\frac{\partial \tau_{y y}}{\partial y}+\frac{\partial \tau_{y z}}{\partial z}=0 \\
\frac{\partial \tau_{z z}}{\partial z}=\rho_{i} g
\end{gathered}
$$

A further approximation, known as the shallow-shelf approximation (SSA), is obtained by neglecting vertical shear (MacAyeal 1992; MacAyeal et al. 1996). This is valid for ice shelves and ice streams characterized by a low basal drag: 


$$
\begin{gathered}
\frac{\partial \tau_{x x}}{\partial x}+\frac{\partial \tau_{x y}}{\partial y}=0, \\
\frac{\partial \tau_{y x}}{\partial x}+\frac{\partial \tau_{y y}}{\partial y}=0, \\
\frac{\partial \tau_{z z}}{\partial z}=\rho_{i} g .
\end{gathered}
$$

For ice streams, an extra basal boundary condition is added, i.e. $\tau_{b}=\beta^{2} u$, where $\tau_{b}$ is the basal shear stress, $\beta^{2}$ is a friction parameter, and $u$ is the horizontal velocity $\left(\beta^{2}=0\right.$ in ice shelves). The basal sliding law can also take a nonlinear form (see below).

The most common approximation is the shallow-ice approximation (SIA). This approximation incorporates only vertical shear stresses, which is valid for an ice mass with a small aspect ratio (i.e. thickness scale much smaller than length scale):

$$
\frac{\partial \tau_{x z}}{\partial z}=\rho_{i} g \frac{\partial s}{\partial x}, \quad \frac{\partial \tau_{y z}}{\partial z}=\rho_{i} g \frac{\partial s}{\partial y},
$$

where $s$ is the surface elevation. Its main advantage is that all stress and velocity components are locally determined. However, the approximation is not valid for key areas such as ice divides and grounding lines (Hutter 1983; Baral et al. 2001).

\subsubsection{Transition Zones}

Besides being the limit of flotation, a grounding line is also the change from a sheardominated ice flow to an ice flow dominated by longitudinal stretching. ${ }^{1}$ The transition is never sharp, but gradual, and knowing the size of this transition zone is essential in understanding grounding line dynamics and inland ice response to sudden changes at the grounding line.

Herterich (1987) calculated the flow within a small transition zone, where the grounded ice sheet is frozen to the bed. The length of the transition zone was found to be of the order of the ice thickness and increased when basal sliding was incorporated. However, the geometry of these experiments was fixed (i.e. no change of the free surface) and the results obtained strongly dependent on this assumption.

Therefore, Lestringant (1994) took in account free-surface changes using a mixed ${ }^{2}$ finite-element method to solve the flow equations within two-dimensional sharp transition zones. He concluded that it was impossible to use reduced Stokes equations in the transition zone and that the ice sheet and ice shelf could be linked by a jump-boundary condition for the horizontal velocity. However, he assumed no drag effect coming from the sides, which means that the ice shelf does not affect the upstream flow.

Similar conclusions were reached by Pattyn (2000), using a two-dimensional flowline model on a fixed finite-difference grid in order to evaluate the impact of model resolution on ice dynamics near the grounding line. He demonstrated that the transition zone is smaller than the grid size at coarse resolutions (i.e. grid sizes of $20-40 \mathrm{~km}$ ). At finer resolutions, the transition zone is larger than the grid size, and hence all stress components

\footnotetext{
${ }^{1}$ This is less valid in the case of an ice stream, where upstream of the grounding line longitudinal stress gradients may be dominant.

2 'Mixed' means that the computed unknowns are the horizontal and vertical velocities as well as pressure (Lestringant 1994).
} 
should be considered. As shown by Pattyn et al. (2006), the transition zone length scale is roughly inverse to basal friction as $0<\beta^{2}<+\infty$. Marine ice sheets with large transition zones (low $\beta^{2}$ values), such as ice streams, seem highly sensitive to perturbations at the grounding line or reduction in buttressing compared to ice sheets with small transition zones (high $\beta^{2}$ ).

Hindmarsh (2004) presented a computational analysis of the accuracy of different approximations to the Stokes equations. He showed that the inclusion of longitudinal (or membrane) stresses increases accuracy at smaller wavelengths compared with the SIA. From his analysis, two longitudinal stress schemes, namely L1L2 (a single-layer scheme, i.e. two-dimensional) and LMLa (multilayer scheme, i.e. three-dimensional; Pattyn 2003), are adequate approximations. LMLa is slightly more accurate than L1L2, but the latter has the advantage that it needs less computational effort since it is two-dimensional. Hindmarsh (2006) proposed a boundary layer in these membrane stresses extending about $10 \mathrm{~km}$ from the grounding line.

The flow of an ice sheet or a glacier is an example of free surface thin film flow, which can be described by two types of models. Lubrication models are appropriate when shear stresses are dominant in the force balance and in the absence of wall slip. Conversely, membrane models are used in the case of dominant normal stresses and rapid wall slip. However, both rapid and slow slip can occur within the same ice mass (e.g. surges, ice streams). Therefore, hybrid lubrication/membrane models, such as HOM (Blatter 1995; Pattyn 2003), have been developed. Schoof and Hindmarsh (2010) established a theory for such hybrid models, which was missing until now. They developed asymptotic expansions for the solution to the Blatter equations in order to obtain a depth-integrated model that describes both fast and slow sliding.

\subsection{Grounding Line Migration}

To accurately capture grounding line migration, it is necessary to resolve the transition zone at a sufficiently fine resolution. Furthermore, besides the flotation criterion (1), an extra boundary condition is needed, i.e. longitudinal stresses should be evaluated at both sides of the grounding line. Schoof (2007a) proposed a semi-analytical solution for the ice flux across the grounding line $q_{g}$ obeying both boundary conditions:

$$
q_{g}=\left[\frac{\bar{A}\left(\rho_{i} g\right)^{n+1}\left(1-\rho_{i} / \rho_{w}\right)^{n}}{4^{n} C}\right]^{\frac{1}{m+1}} \theta^{\frac{n}{m+1}} h_{g}^{\frac{m+n+3}{m+1}},
$$

where $\bar{A}$ is the depth-averaged parameter in Glen's flow law, $n$ is the Glen's flow law exponent, $C$ is the basal sliding parameter, $m$ is the basal sliding exponent, $\theta$ is the buttressing factor ( $\theta=1$ in this study), and $h_{g}$ is the ice thickness at the grounding line. This implies that ice flux at the grounding line is sensitive to changes in the ice shelf, contrary to earlier findings by Hindmarsh (1993).

Pollard and DeConto (2009) incorporated this solution in a numerical ice sheet model at coarse grid resolution by applying a heuristic rule: if the semi-analytical derived flux across the actual grounding line $q_{g}$ from (13) is greater than the modeled flux through the last grounded grid point $q_{i}$, then $q_{g}$ is imposed at that grid point. The velocity at the last grounded grid point $u_{i}$ is calculated by dividing the analytical flux $q_{g}$ by the ice thickness at that grid point $h_{i}$ (derived from the numerical advection scheme). Otherwise, $q_{g}$ is imposed one grid point further downstream (i.e. the first floating grid point) and the velocity at the 
Fig. 1 Fixed grid heuristic rule adapted from Pollard and DeConto (2009)

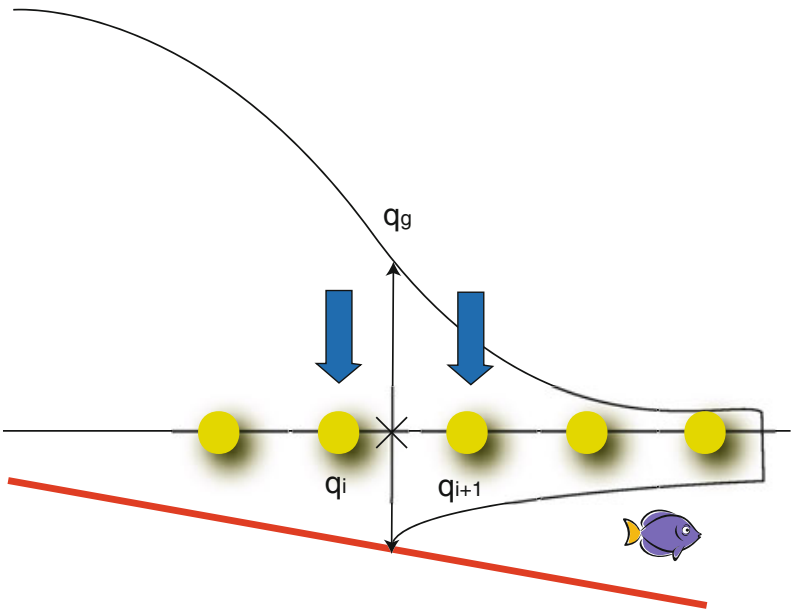

first floating grid point $u_{i+1}$ equals the analytical flux $q_{g}$ divided by the ice thickness there $h_{i+1}$. The former is usually associated with grounding line retreat, and the latter usually with grounding line advance (Fig. 1):

$$
\begin{gathered}
q_{g}>q_{i}: q_{i}=q_{g} \quad \text { or } \quad u_{i}=\frac{q_{g}}{h_{i}}, \\
q_{g}<q_{i}: q_{i+1}=q_{g} \quad \text { or } \quad u_{i+1}=\frac{q_{g}}{h_{i+1}} .
\end{gathered}
$$

The reason why the flux is imposed (and not the velocity) is that the equilibrated solutions in a finite-difference model have the same property as the semi-analytical Schoof solutions: namely, that the upstream snowfall integrated from the grounding line to the ice divide equals the flux across the grounding line. And since the latter is a function of the grounding line ice thickness (Schoof 2007a), which in turn depends on the grounding line position (for a given sea level and bed profile), the equilibrium grounding-line position is determined by the above balance, and is independent of all other model dynamics (as long as the model conserved mass).

Both principles (Schoof boundary condition and Pollard and DeConto heuristic rule) form the base of a simple ice sheet model developed in the next section.

\section{A Simple Grounding Line Model}

\subsection{Model Description}

The numerical ice sheet model used here is a one-dimensional (vertically integrated) finitedifference flowline model. The grounded part is either based on the shallow-ice approximation (thereby including vertical shear stresses) or the shallow-shelf model (with inclusion of basal friction, i.e. a so-called L1L2 model). In any case, the floating part is according to the shallow-shelf approximation. Therefore, two models are used here, namely SIA/SSA (SIA for the sheet and SSA for the shelf) and SSA (SSA for the whole domain). The depth-averaged horizontal velocity in the ice sheet is calculated as follows: 


$$
\bar{u}=u_{b}+\frac{2}{n+2} \bar{A} h\left|\tau_{d}\right|^{n-1} \tau_{d}
$$

where $\tau_{d}=-\rho_{i} g h \nabla s$ is the driving stress ( $\tau_{d} \equiv \tau_{b}$ in the case of SIA). Basal velocity $u_{b}$ is derived from a Weertman-type sliding law:

$$
u_{b}=C^{-\frac{1}{m}}\left|\tau_{d}\right|^{\frac{1}{m}-1} \tau_{d} .
$$

The shallow-shelf (SSA) model neglects vertical shearing and ice deformation is dominated by membrane stresses:

$$
4 \frac{\partial}{\partial x}\left\{\eta \frac{\partial \bar{u}}{\partial x}\right\}+\beta^{2} u_{b}=\rho_{i} g \frac{\partial s}{\partial x}
$$

where

$$
\eta=\frac{1}{2} \bar{A}^{-\frac{1}{n}}\left\{\frac{\partial \bar{u}}{\partial x}\right\}^{\frac{1-n}{n}}
$$

is the effective viscosity. Due to its depth-integrated nature, it follows that $u_{b} \equiv \bar{u}$ in (18). The basal friction parameter $\beta^{2}$ is then defined as:

$$
\beta^{2}=\left\{\begin{array}{ccc}
C|\bar{u}|^{m-1} & : & x<x_{g} \\
0 & : & x \geq x_{g}
\end{array}\right.
$$

where $x_{g}$ is the position of the grounding line. A symmetric ice divide is considered the upstream boundary:

$$
\frac{\partial(h+b)}{\partial x}=0 ; \quad \bar{u}=0
$$

At the downstream boundary (ice shelf/ocean), the longitudinal stress gradient is balanced by the hydrostatic pressure of the ocean water (Paterson 1994):

$$
\frac{\partial \bar{u}}{\partial x}=\bar{A}\left[\frac{1}{4} \rho g h\left(1-\frac{\rho_{i}}{\rho_{w}}\right)\right]^{n} .
$$

The mass conservation equation is integrated along the vertical to obtain the ice thickness evolution:

$$
\frac{\partial h}{\partial t}+\frac{\partial(\bar{u} h)}{\partial x}=\dot{a},
$$

where $\dot{a}$ is the accumulation rate.

There are several ways in which grounding line migration can be treated in a finitedifference model (Gladstone et al. 2010). One way is to determine the grounding line position $x_{g}$ by linear interpolation between the last grounded grid point position $x_{i}$ and the first floating point $x_{i+1}$ using the flotation criterion expressed in terms of height above flotation $h^{*}$, i.e.

$$
x_{g}=x_{i}-\frac{h_{i}^{*}}{\nabla h^{*}},
$$

where 


$$
\begin{gathered}
h_{i}^{*}=b_{i}-z_{s l}+h_{i} \frac{\rho_{i}}{\rho_{w}}, \\
\nabla h^{*}=\frac{h_{i+1}^{*}-h_{i}^{*}}{\Delta x},
\end{gathered}
$$

and where $\Delta x$ is the grid size. Ice thickness at the grounding line $h_{g}$ is then linearly interpolated from its known position $x_{g}$. We enabled grounding line migration using (13) combined with the Pollard and DeConto heuristic rule (14) and (15).

\subsection{Numerical Implementation}

The model was implemented on a fixed finite-difference grid, both in a staggered and a non-staggered version. For the staggered model, velocities are determined between grid points ( $u$-grid), while for the non-staggered version velocities are calculated on the grid points where the ice sheet geometry is available ( $h$-grid). The mass conservation equation (21) is discretized using a semi-implicit scheme and coded as FTCS (forward in time, central in space). This gives for a staggered and a non-staggered grid, respectively

$$
\begin{gathered}
h_{i, t+1}+\frac{\Delta t}{2 \Delta x}\left[u_{i+\frac{1}{2}}\left(h_{i+1, t+1}+h_{i, t+1}\right)-u_{i-\frac{1}{2}}\left(h_{i-1, t+1}+h_{i, t+1}\right)\right]=h_{i, t}+\dot{a} \Delta t \\
h_{i, t+1}+\frac{\Delta t}{2 \Delta x}\left[u_{i+1} h_{i+1, t+1}-u_{i-1} h_{i-1, t+1}\right]=h_{i, t}+\dot{a} \Delta t
\end{gathered}
$$

where indices $i=1: N$ are the grid nodes along the flowline, and index $t$ denotes time. For the SIA model, (25-26) are written as a diffusive equation [see Huybrechts et al (1996) for a more detailed description and discussion]. However, the combined diffusive-advective equations lead to mass loss across the grounding line with the non-staggered model, which is not the case with the staggered model, where mass is always conserved along the whole flowline irrespective of the physical model (SIA/SSA or SSA). The effect of mass loss across the grounding line is discussed in Pattyn et al. (2006) and has been shown to have no qualitative impact on grounding line migration.

\subsection{Experiments}

Vieli and Payne (2005) showed that marine ice sheet FG models were highly sensitive to horizontal grid size. Large grid sizes, for instance, prevent grounding line migration. Only small enough grid sizes lead to grounding line advance (Huybrechts et al. 1998; Vieli and Payne 2005; Durand et al. 2009b). To test the grid size sensitivity, we carried out model experiments with different grid sizes, i.e. 50, 25, 12.5, and $5 \mathrm{~km}$ as well as for different physical approximations (SIA/SSA and SSA) and numerical approaches (staggered and non-staggered grids). We compared our model results to the semi-analytical solution derived by Schoof (2007).

The first experiment is a MISMIP variant ${ }^{3}$ (Schoof et al. 2007). A steady state geometry is developed on a linearly downward-sloping bedrock defined by

$$
b(x)=-100-x,
$$

\footnotetext{
${ }^{3}$ MISMIP: Marine Ice Sheet Model Intercomparison Project; http://homepages.ulb.ac.be/ fpattyn/mismip/
} 
Table 1 Constants and parameter settings for the first, third and fourth experiments

\begin{tabular}{llll}
\hline Parameter & Description & Value & Unit \\
\hline$\rho_{i}$ & Ice density & 900 & $\mathrm{~kg} \mathrm{~m}^{-3}$ \\
$\rho_{w}$ & Water density & 1,000 & $\mathrm{~kg} \mathrm{~m}^{-3}$ \\
$g$ & Gravitational acceleration & 9.8 & $\mathrm{~m} \mathrm{~s}^{-2}$ \\
$n$ & Glen's flow law exponent & 3 & \\
$a$ & Accumulation rate & 0.3 & $\mathrm{~m} \mathrm{a}^{-1}$ \\
$L$ & Domain length & 1,000 & $\mathrm{~km}^{\prime}$ \\
$C$ & Basal friction coefficient & 107 & $\mathrm{~Pa} \mathrm{~s}^{m} \mathrm{~m}^{-m}$ \\
$m$ & Basal friction exponent & $1 / 3$ & \\
\hline
\end{tabular}

where $b$ is bedrock elevation (m a.s.l.) and $x$ is distance from the ice divide ( $\mathrm{km})$. Other parameters and constants are given in Table 1. In the standard experiment, a value for Glen's flow law parameter of $A=10^{-25} \mathrm{~Pa}^{-n} \mathrm{~s}^{-1}$ is used. A steady state is achieved after $\sim 40,000$ years of integration. Starting from this steady state configuration (further addressed to as initial state), the value for $A$ is decreased (increasing viscosity), leading to an advance of the grounding line. This process is repeated for subsequent changes in $A$, ranging from $10^{-25}$ to $10^{-26}$ in steps of $2 \times 10^{-26}$, as well as the reverse process (starting from $A=10^{-26}$ and decreasing the viscosity in steps of $2 \times 10^{-26}$ ). Each of these step changes takes $\sim 20,000$ years to reach a steady state. What is referred to below as final state is the steady state after complete advance and retreat and for the value of $A=10^{-25} \mathrm{~Pa}^{-n} \mathrm{~s}^{-1}$.

The second experiment corresponds to MISMIP Experiment 3a, where a steady state geometry is allowed to develop on an overdeepened polynomial bedrock, defined by

$$
b(x)=729-2184.8 \times\left(\frac{x}{750 \mathrm{~km}}\right)^{2}+1031.72 \times\left(\frac{x}{750 \mathrm{~km}}\right)^{4}-151.72 \times\left(\frac{x}{750 \mathrm{~km}}\right)^{6} .
$$

The flow parameter $A$ is varied stepwise between $3 \times 10^{-25}$ and $2.5 \times 10^{-26} \mathrm{~Pa}^{-n} \mathrm{~s}^{-1}$. Other parameters are similar to those of the previous experiment, except for the domain length, taken as $L=1800 \mathrm{~km}$ and the basal sliding parameter set to $C=7.624 \times 10^{6} \mathrm{~Pa}$ $\mathrm{s}^{m} \mathrm{~m}^{-m}$.

In the third experiment, we used the same linearly downward-sloping bed (27) and same constants as in the first experiment above (Table 1). We started from an initial steady state ice sheet with $A=10^{-25} \mathrm{~Pa}^{-n} \mathrm{~s}^{-1}$. The bulk viscosity of the ice sheet was increased by setting $A=4 \times 10^{-26} \mathrm{~Pa}^{-n} \mathrm{~s}^{-1}$, leading to a grounding line advance, until a new steady state was reached. Subsequently, the viscosity was decreased by setting $A=10^{-25} \mathrm{~Pa}^{-n}$ $\mathrm{s}^{-1}$, invoking a grounding line retreat to the initial state.

A major drawback of the Schoof solution is its validity restricted to steady state solutions and not to transient states. However, grounding line migration rate is a major issue when trying to understand marine ice sheet response over decadal time scales (as needed in IPCC projections). The transient response of the grounding line should therefore be independent of numerical parameters, such as grid resolution and time step, and should be coherent with theoretical developments. Therefore, we performed a fourth experiment to investigate the time-dependent response by evaluating ice fluxes across the grounding line with theoretical values (i.e. Schoof solution) and grounding line migration rate during grounding line advance and retreat for a staggered SSA model. We used the same setup and parameters as the third experiment above (i.e. linearly downward-sloping bed), except 
that we used 5 values of rate factor $A$ instead of 3 in order to compare the transient behavior with the same viscosity for the advance and the retreat. These values are, respectively, $10^{-25}, 4 \times 10^{-26}, 10^{-26}, 4 \times 10^{-26}$ and $10^{-25} \mathrm{~Pa}^{-n} \mathrm{~s}^{-1}$.

\section{Results}

\subsection{Effect of Grid Resolution on Steady State Grounding Line Position}

\subsubsection{Downward-Sloping Bed}

Figure 2 shows the steady state profiles of the ice sheet and ice shelf along the flowline for both the linearly downward-sloping and the overdeepened bedrock profiles (staggered SSA model). Grounding line advance is obtained when decreasing the value of $A$, and a retreat is invoked when $A$ is increased to its initial value. Advance and retreat steady state profiles are hardly discernible, as both are lying close together. According to theory (Schoof 2007a), they should overlap, which defies neutral equilibrium of grounding line positions.

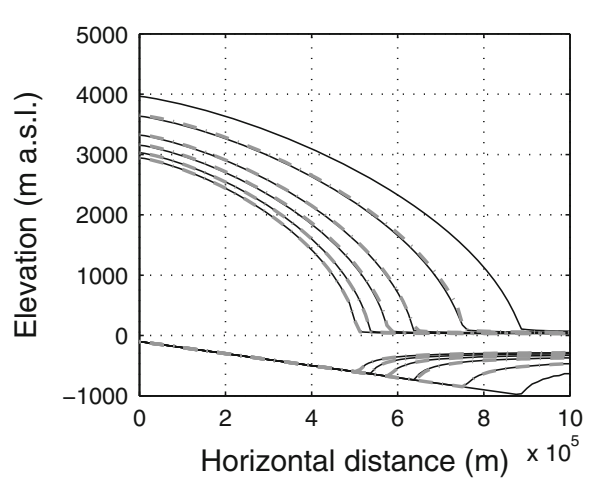

(a) Downward-sloping bed

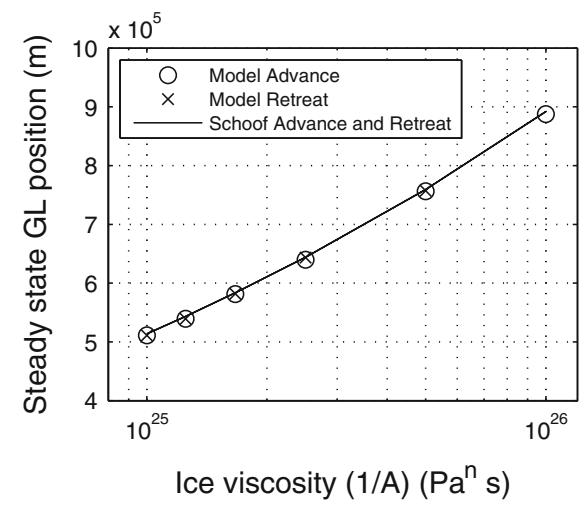

(c) Downward-sloping bed

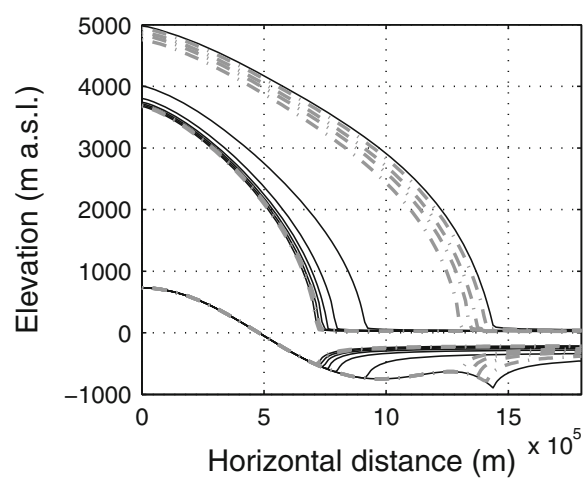

(b) Overdeepened bed

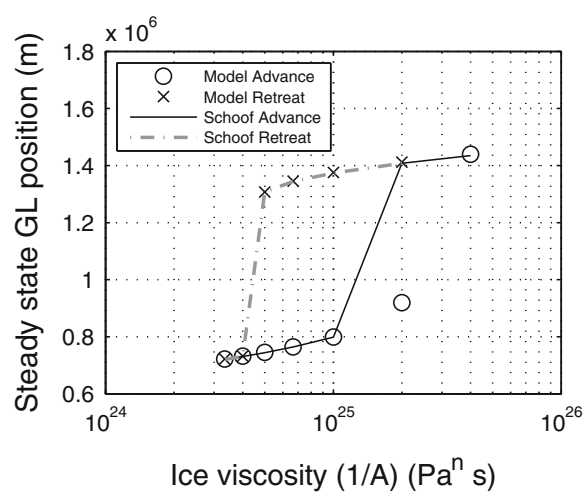

(d) Overdeepened bed

Fig. 2 Steady state profiles (solid black curve advance; dashed gray curve retreat) of the ice sheet on a downward-sloping bed (a and $\mathbf{c}$ ) and on an overdeepened bed (b and $\mathbf{d}$ ) for the SSA model on a staggered grid (first and second experiments). Grid size is $12.5 \mathrm{~km}$ in both cases. GL means 'grounding line' 
When analyzing the differences between final (after perturbation of $A$ ) and initial steady state grounding line positions as well as the differences between final steady state grounding line positions and Schoof semi-analytical solution for the whole suite of grid resolutions as well as for the different physical and numerical approximations, it becomes clear that staggered grid models generally perform well in reproducing advance and retreat of the grounding line with high accuracy (Table 2). Especially the SSA staggered grid model displays differences between grounding line positions of the order of meters to tens of meters, which is a fraction of the grid size. Furthermore, there is no apparent relation between the accuracy and grid resolution. The same conclusions can be drawn for the SIA/ SSA staggered grid model, albeit that differences are an order of magnitude larger, but still small compared to the grid size. The non-staggered grid model, on the contrary, shows the largest discrepancies in which differences are of the order of magnitude of the grid size (and therefore decreasing with decreasing grid size). Similar tests were also performed with staggered SSA and SIA/SSA models that do not include the Schoof boundary condition (not shown). They also reveal large discrepancies between the advance and retreat steady state positions and in the majority of the experiments the grounding line hardly retreats when $A$ is set to its initial value.

In summary, all staggered grid models that are forced with the Schoof boundary condition converge to the same steady state grounding line position irrespective of the horizontal grid size used. Models that are either non-staggered or do not include the boundary condition converge only to the same advance-retreat position for sufficiently small grid sizes, which is considered a deficiency of fixed grid finite-difference models (Vieli and Payne 2005; Durand et al. 2009b; Gladstone et al. 2010). Results also depend on model type, i.e. the SSA model gives better results (difference between final and initial grounding line positions is less) compared to SIA/SSA (Table 2).

Table 2 Absolute differences between final (after perturbation of $A$ ) and initial steady state grounding line (GL) positions $\left(\Delta F I=\left|G L_{\text {Final }}-G L_{\text {Initial }}\right|\right)$ and absolute differences between final steady state GL positions and Schoof semi-analytical solution $\left(\Delta F S=\left|G L_{\text {Final }}-G L_{\text {Schoof }}\right|\right)$ as a function of grid size for the SSA staggered grid model, SIA/SSA staggered grid model and SIA/SSA non-staggered grid model

\begin{tabular}{llllcr}
\hline Physical approx. & Grid type & Grid size $(\mathrm{m})$ & Runs & $\Delta F I(\sigma)(\mathrm{m})$ & $\Delta F S(\sigma)(\mathrm{m})$ \\
\hline SSA & Staggered & 50000 & 4 & $3.17(3.74)$ & $7020(3018)$ \\
SSA & Staggered & 25000 & 4 & $28.01(38.05)$ & $4567(1287)$ \\
SSA & Staggered & 12500 & 4 & $6.54(9.27)$ & $3194(793)$ \\
SSA & Staggered & 5000 & 2 & $0.94(0.32)$ & $2015(231)$ \\
SIA/SSA & Staggered & 50000 & 2 & $127.39(116.48)$ & $9616(1405)$ \\
SIA/SSA & Staggered & 25000 & 2 & $165.26(36.83)$ & $5750(476)$ \\
SIA/SSA & Staggered & 12500 & 1 & $65.57(0)$ & $2905(0)$ \\
SIA/SSA & Non-staggered & 50000 & 5 & $58610(15388)$ & $73243(8378)$ \\
SIA/SSA & Non-staggered & 25000 & 5 & $25901(7974)$ & $54154(4602)$ \\
SIA/SSA & Non-staggered & 12500 & 5 & $8983(2367)$ & $43904(2365)$ \\
SIA/SSA & Non-staggered & 5000 & 5 & $1413(1144)$ & $37529(712)$
\end{tabular}

Instead of performing only one model run per grid size, we performed a series of model runs for a 'nominal' grid size in which the initial grid size was slightly altered by adding/subtracting 1 or 2 grid points for the whole flowline, thereby keeping the flowline length constant. Standard deviation $(\sigma)$ is indicated in brackets. These results correspond to the third experiment (linearly downward-sloping bedrock and $3 A$ values) 
Even though modeled grounding line position is unique for each set of parameter values, deviations from the Schoof semi-analytical solution may occur. These differences decrease with decreasing grid size (Table 2). Since the Schoof semi-analytical solution is based on boundary layer theory, these positions do not necessarily have to coincide with the steady state positions for the different model types, as other physics are involved. Nevertheless, the difference is not more than a couple of kilometers for the staggered grid models, since all models are forced with the boundary condition from Schoof (2007a). In concordance with the above results, SIA/SSA models show a larger deviation from the Schoof solution and the non-staggered model deviates with several tens of kilometers.

\subsubsection{Overdeepened Bed}

For an overdeepened bed, multiple steady states occur for the same value of $A$ as a function of the initial geometry of the ice sheet. This is due to the fact that no steady states are found on upward-sloping beds (Schoof 2007a), leading to hysteresis (Fig. 2b, d). In general, conclusions reached in "Subsect. 4.1.1" are also valid here, i.e. initial and final grounding line positions show differences of the order of meters to tens of meters with the staggered SSA model, while large deviations - also related to grid size, occur for either SIA/SSA and non-staggered grid models (not shown).

\subsection{Grounding Line Migration Rate}

Figure 3 displays the grounding line flux as a function of time during the advance (Fig. 3a) and the retreat (Fig. 3b) compared to the flux determined from (13). Since the value of rate factor $A$ is the same for both advance and retreat, the ice flux converges towards the same value in Fig. 3a and b. Both solutions show a series of distinct jumps in time that are mainly due to the intrinsic discrete nature of the heuristic rule in (14-15), which causes flips between grid points for arbitrarily small changes of ice flux. These discrete steps cannot be removed despite the interpolating grounding line function (22). For an advancing ice sheet, the difference between the modeled and semi-analytical fluxes is relatively small. However, during retreat sudden high amplitude increases in grounding line flux occur with the SSA model. The peaks can be visualized in more detail in Fig. 3d, where ice fluxes are plotted against grounding line ice thickness. Nevertheless, after these jumps the modeled fluxes converge toward the fluxes determined from (13). Since the Schoof flux given by (13) is valid for steady state conditions and derived from boundary layer theory, deviations from this semi-analytical flux during a transient state do not indicate whether the model results are pertinent or not.

A sound way of evaluating grounding line migration rate is to compare the migration rate obtained with the model with the one calculated from differentiating the flotation criterion (Hindmarsh 1993), i.e.

$$
\frac{d x_{g}}{d t}=-\frac{\dot{a}-\frac{\partial q}{\partial x}}{\frac{\partial h}{\partial x}+\frac{\rho_{w}}{\rho_{i}} \frac{\partial b}{\partial x}}
$$

We solved (29) numerically by calculating both the flux divergence and the thickness gradient with a three-point upstream difference scheme centered on the sub-grid grounding line position $x_{g}$ (Fig. $3 \mathrm{e}$ and f). A distinction is made based on the heuristic rule (14) or (15). Circles in the scatterplot correspond to the case where the grounding line flux is larger than the flux obtained from (13), generally leading to a grounding line advance. Crosses 


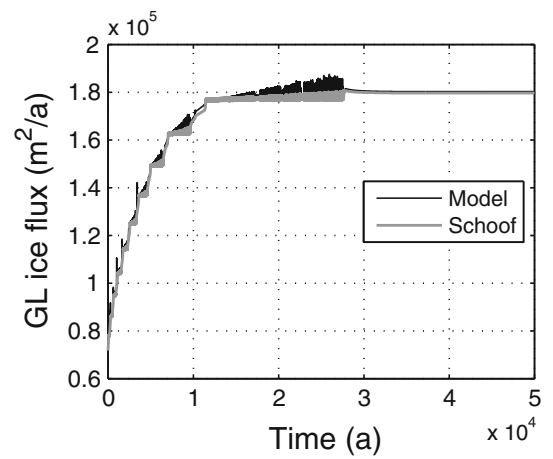

(a) Advance

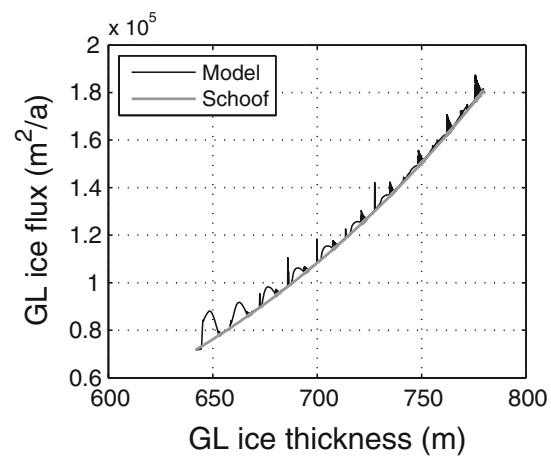

(c) Advance

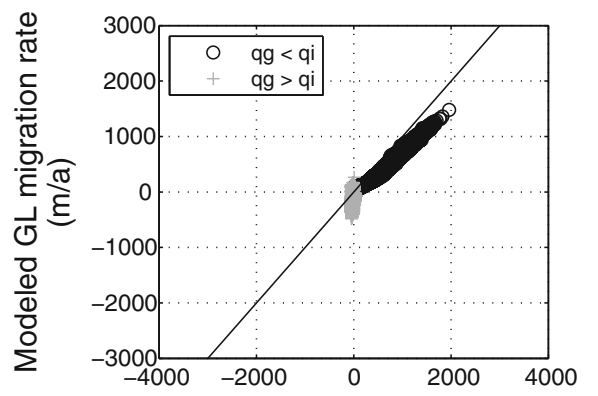

GL migration rate given by (29) (m/a)

(e) Advance

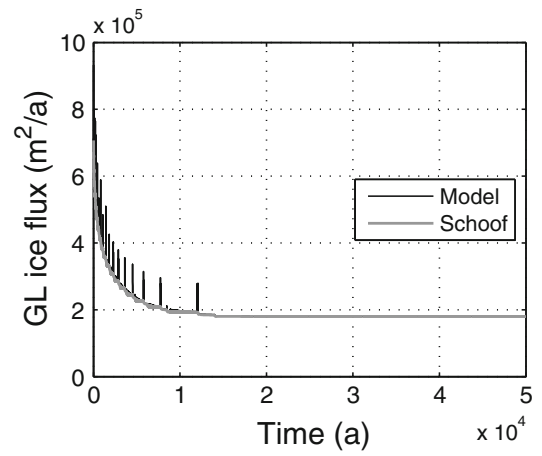

(b) Retreat

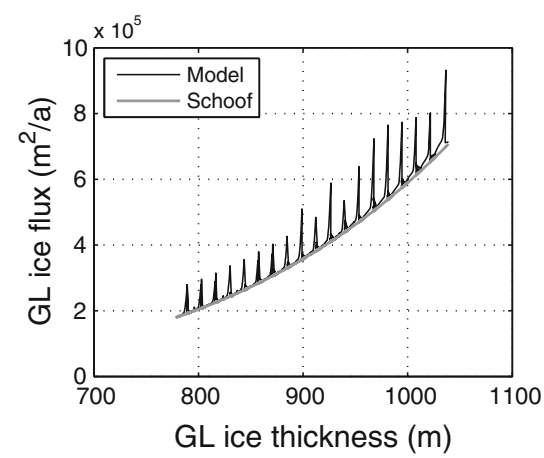

(d) Retreat

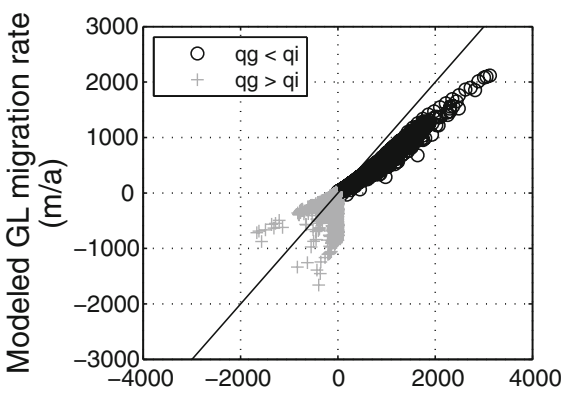

GL migration rate given by (29) (m/a)

(f) Retreat

Fig. 3 Modeled grounding line (GL) flux compared to Schoof GL flux as a function of time (a and b) and GL ice thickness (c and d; fourth experiment). Modeled GL migration rate $\left(d x_{g} / d t\right)$ is also plotted against GL migration rate given by (29). Left plots show the advance stage ( $A$ decreases from $10^{-25}$ to $4 \times 10^{-26}$ $\left.\mathrm{Pa}^{-n} \mathrm{~s}^{-1}\right)$, while right plots show the retreat stage $\left(A\right.$ increases from $10^{-26}$ to $\left.4 \times 10^{-26} \mathrm{~Pa}^{-n} \mathrm{~s}^{-1}\right)$. Grid size is $12.5 \mathrm{~km}$

correspond to the inverse case [grounding line flux smaller than the flux obtained from (13)]. For both advance and retreat experiments, grounding line changes in either direction occur, albeit that negative migration rates are generally absent for the advancing case (Fig. 3e). 
In general there are many more 'retreat' points (crosses) than there are 'advance' points (circles). The heuristic rule compares the modeled ice flux to the theoretical flux and adapts the modeled velocity field accordingly. Any 'advance' flux condition will alter the flow field considerably, and result in an important change in grounding line position as well as in glacier geometry. However, during the following time steps the flux at the grounding line will be too low compared to the theoretical one and slight adjustments to the jump in grounding line position are made (retreat), to compensate for the larger initial jump. These changes happen evidently on sub-grid level, leading to a negative migration rate. Therefore, during an advance phase, small 'retreat' changes can be observed, simply to counterbalance large changes due to the imposition of the grounding line flux. This also explains why during an advance phase small episodes of grounding line retreat can be observed, while according to theory, this should be zero (Fig. 3e). Both Fig. 3e and f show that modeled advance rates correspond well with the theoretical value, but are slightly underestimated, while the modeled retreat rates show a larger discrepancy, probably related to this so-called counterbalancing effect.

A similar experiment was carried out for a smaller grid size $(6.25 \mathrm{~km}$, Fig. 4). The behavior is coherent in the sense that, due to the higher resolution, jumps in grounding line flux occur more often, but they are of smaller amplitude. The comparison of modeled grounding line migration rates with the calculated ones leads to a similar graph as in the previous experiment. Migration rates are comparable and invariant for the grid size applied, albeit slightly lower than those determined from (29).

\section{Discussion and Outlook}

Representing grounding line migration in numerical ice sheet models remains an arduous task, not only because of the complexity of the physics involved (change from shear dominated flow to flow dominated by longitudinal pulling or stretching), the geometry of the problem (a grounding line is difficult to identify within a numerical model as it does not always coincide with a grid node), the computational challenge (solving a complex set of equations on a high-resolution mesh), but also the difficulty to observe and attribute the process of grounding line migration due to different interacting mechanisms, such as subshelf melting, loss of buttressing, inland ice flow acceleration due to basal sliding, ice thinning, etc. All these processes may cause grounding line retreat, but a number of them are at the same time a result of grounding line retreat as well, hence leading to a series of feedbacks. Therefore, observations alone are not sufficient to disentangle the mechanisms involved. Nowadays, the gap in our understanding has been filled by advances in timedependent observations, numerical modeling and tools for model verification.

Only recently the debate on grounding line stability has moved onto the next level with the mathematical proof on grounding line steady state position as a function of ice flux and topography (Schoof 2007a), leading to a verification and intercomparison exercise for numerical ice sheet models (Schoof et al. 2007). In this paper we compared numerical results obtained with simple ice sheet models against the semi-analytical solutions proposed by Schoof et al. (2007), revealing the necessity of having numerical models with sound numerical treatments and proper physical approximations. Although the semi-analytical solution is only valid for steady states, the transient behavior of the model has been analyzed by different means.

The results presented in this paper are obtained with a couple of simple ice sheet/ice shelf models that capture the essence of grounding line migration by including the heuristic 


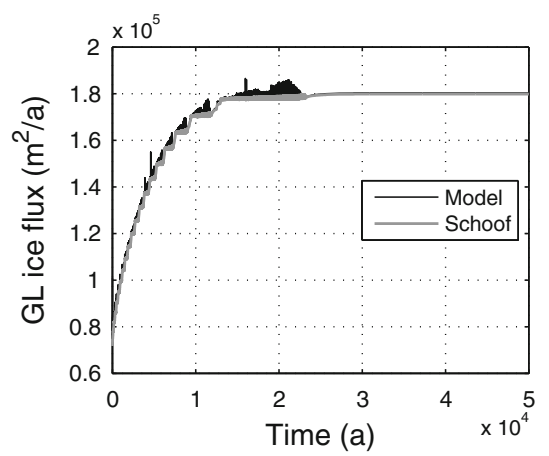

(a) Advance

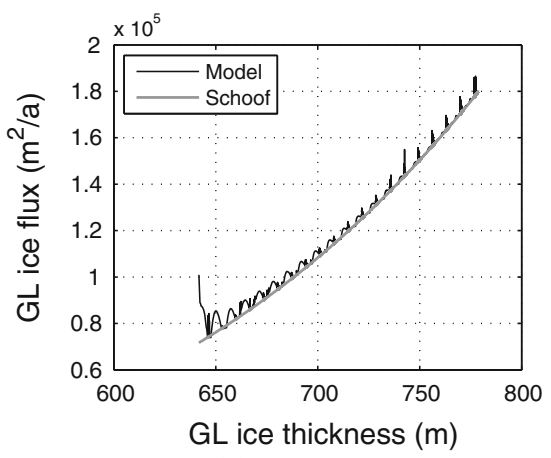

(c) Advance

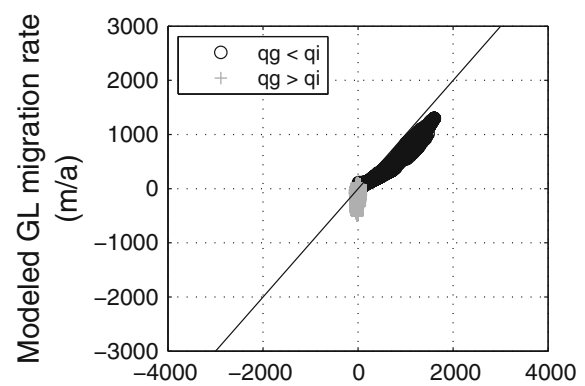

GL migration rate given by (29) $(\mathrm{m} / \mathrm{a})$

(e) Advance

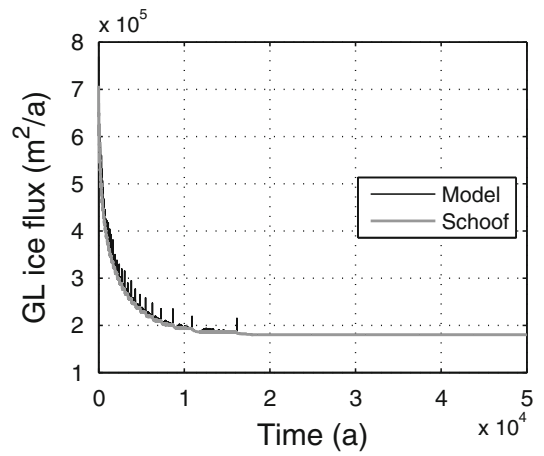

(b) Retreat

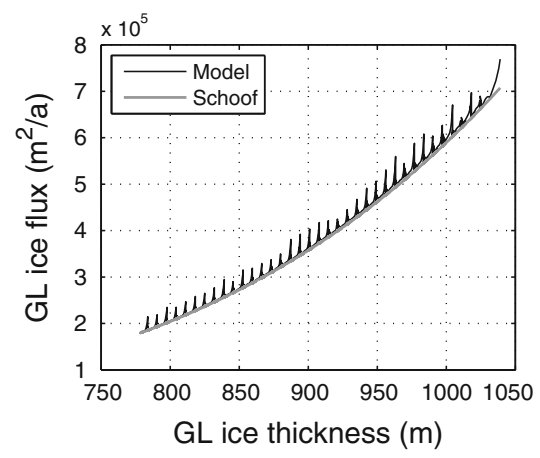

(d) Retreat

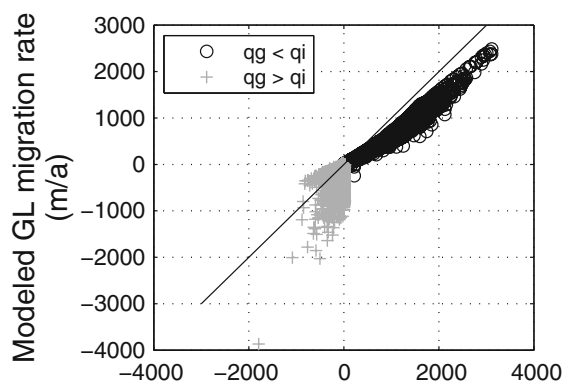

GL migration rate given by $(29)(\mathrm{m} / \mathrm{a})$

(f) Retreat

Fig. 4 Same as figure 3 with a grid size of $6.25 \mathrm{~km}$

rule proposed by Pollard and DeConto (2009). The latter assures that modeled grounding line positions are in accord with the steady state grounding line positions given by Schoof (2007a). Ice sheet models on a staggered grid perform well and lead to high accuracy on steady state grounding line position (comparing advancing to retreating ice sheets). Nonstaggered grid models lead to larger differences and accuracy increases with decreasing grid size. This poorer performance may be related to mass loss occurring at the grounding line while coupling a diffusive with an advective scheme, as discussed in Pattyn et al. (2006). 
The time-dependent response of these models remains influenced by the parameterization scheme of grounding line migration, leading to sudden changes in grounding line ice flux whenever a jump from one grid point to another occurs, despite the sub-grid representation of grounding line position in the model. However, the bulk response in grounding line migration rate is coherent when compared with those expected from differentiating the flotation criterion at the grounding line (Hindmarsh 1993), albeit that modeled migration rates are generally underestimated.

A major drawback of the models presented in this paper is their limitation along a flowline. Although buttressing effects can be included in a parameterized way (Vieli and Payne 2005; Pattyn et al. 2006), real three-dimensional effects are lacking. A number of three-dimensional models that cope with grounding line migration in a verified way have been developed or are in the process of development (Pollard and DeConto 2009; Goldberg et al. 2009; Robison et al. 2009; Katz and Worster 2010), where the Pollard and DeConto model incorporates the heuristic rule as in the above experiments. A more detailed comparison with higher-order and Full Stokes models should be carried out in order to remove any bias towards the use of such types of models. International efforts, such as ice 2 sea $^{4}$ or SeaRISE, ${ }^{5}$ are pushing the ice sheet model community to developing better and physicallybased numerical ice sheet models for future sea-level change projections. Once such models are fully available, a physically-based assessment will be possible, making the use of selective grounding line migration scenarios obsolete.

Acknowledgments This work was supported by both the IceCube-Dyn project (Actions de recherche concertées) funded by the French Community of Belgium and the ice2sea project funded by the European Commission's 7th Framework Programme through grant 226375 (ice2sea manuscript No. 20). The authors are greatly indebted to Gael Durand for his constructive review and to David Pollard who pointed out an error in the initially submitted manuscript.

\section{References}

Baral DR, Hutter K, Greve R (2001) Asymptotic theories of large-scale motion, temperature, and moisture distribution in land-based polythermal ice sheets: a critical review and new developments. Appl Mech $\operatorname{Rev} 54(3): 215-256$

Blatter H (1995) Velocity and stress fields in grounded glaciers: a simple algorithm for including deviatoric stress gradients. J Glaciol 41(138):333-344

Chen JL, Wilson CR, Blankenship D, Tapley BD (2009) Accelerated Antarctic ice loss from satellite gravity measurements. Nat Geosci 2:859-862

Durand G, Gagliardini O, de Fleurian B, Zwinger T, Meur EL (2009a) Marine ice sheet dynamics: hysteresis and neutral equlibrium. J Geophys Res 114(F03009):170. doi:10.1029/2008JF001

Durand G, Zwinger T, Meur EL, Hindmarsh RCA (2009b) Full stokes modeling of marince ice sheets: influence of the grid size. Ann Glac 50(2)

Gladstone RM, Payne AJ, Cornford SL (2010) Parameterising the grounding line in ice sheet models. Cryosphere Discuss 4:1063-1105

Goldberg D, Holland DM, Schoof C (2009) Grounding line movement and ice shelf buttressing in marine ice sheets. J Geophys Res 114(F024026):227. doi:10.1029/2008JF001

\footnotetext{
4 ice2sea is a science programme that is funded by the European Union Framework-7 scheme and will improve projections of the contribution of ice to future sea-level rise, http://www.ice2sea.eu.

5 SeaRISE (Sea-level Response to Ice Sheet Evolution) is a community organized effort to estimate the upper bound of ice sheet contributions to sea level in the next 100-200 years, http://websrv.cs.umt.edu/isis/ index.php/SeaRISE_Assessment.
} 
Herterich K (1987) On the flow within the transition zone between ice sheet and ice shelf. In: Veen C, Oerlemans J (eds) Dynamics of the west Antarctic ice sheet. Kluwer Academic Publishers, Dordrecht, pp 185-202

Hindmarsh RCA (1993) Qualitative dynamics of marine ice sheets. In: Peltier W (ed) Ice in the climate system, NATO ASI series I, vol 12. Springer, Berlin, pp 67-99

Hindmarsh RCA (1996) Stability of ice rises and uncoupled marine ice sheets. Ann Glaciol 23:105-115

Hindmarsh RCA (2004) A numerical comparison of approximations to the Stokes equations used in ice sheet and glacier modeling. J Geophys Res 109(F01012):065. doi:10.1029/2003JF000

Hindmarsh RCA (2006) The role of membrane-like stresses in determining the stability and sensitivity of the Antarctic ice sheets: back pressure and grounding line motion. Phil Trans R Soc A 364:1733-1767

Hindmarsh RCA, Le Meur E (2001) Dynamical processes involved in the retreat of marine ice sheets. J Glaciol 47(157):271-282

Hutter K (1983) Theoretical glaciology. Kluwer Academic Publishers, Dordrecht

Huybrechts P (1990) A 3-D model for the Antarctic ice sheet: a sensitivity study on the glacial-interglacial contrast. Climate Dyn 5:79-92

Huybrechts P, Payne A, TheEISMINT Intercomparison Group (1996) The EISMINT benchmarks for testing ice-sheet models. Ann Glaciol 23:1-12

Huybrechts P, Abe-Ouchi A, Marsiat I, Pattyn F, Payne A, Ritz C, Rommelaere V (1998) Report of the third EISMINT workshop on model intercomparison. European Science Foundation, Strasbourg

Jenkins A, Dutrieux P, Jacobs S, McPhail S, Perrett J, Webb A, White D (2010) Observations beneath Pine Island Glacier in West Antarctica and implications for its retreat. Nat Geosci 3(7):468-472

Joughin I, Rignot E, Rosanova CE, Lucchitta BK, Bohlander J (2003) Timing of recent accelerations of Pine Island Glacier, Antarctica. Geophys Res Lett 30(13):609. doi:10.1029/2003GL017

Katz RF, Worster MG (2010) Stability of ice-sheet grounding lines. Proc R Soc A. doi:10.1098/ rspa.2009.0434

Lestringant R (1994) A two-dimensional finite-element study of flow in the transition zone between an ice sheet and an ice shelf. Ann Glaciol 20:67-72

MacAyeal DR (1992) Irregular oscillations of the West Antarctic ice sheet. Nature 359:29-32

MacAyeal DR, Rommelaere V, Huybrechts P, Hulbe CL, Determann J, Ritz C (1996) An ice-shelf model test based on the Ross ice shelf. Ann Glaciol 23:46-51

Paterson WSB (1994) The physics of flaciers, 3rd edn. Pergamon Press, Oxford

Pattyn F (2000) Ice-sheet modelling at different spatial resolutions: focus on the grounding line. Ann Glaciol 31:211-216

Pattyn F (2003) A new 3D higher-order thermomechanical ice-sheet model: basic sensitivity, ice-stream development and ice flow across subglacial lakes. J Geophys Res 108(B8, 2382):329. doi:10.1029/ 2002JB002

Pattyn F, Huyghe A, De Brabander S, De Smedt B (2006) Role of transition zones in marine ice sheet dynamics. J Geophys Res 111(F02004):394. doi:10.1029/2005JF000

Pollard D, DeConto RM (2009) Modelling West Antarctic ice sheet growth and collapse through the past five million years. Nature 458:809. doi:10.1038/nature07

Rignot E, Bamber JL, van den Broeke MR, Davis C, Li Y, van de Berg WJ, van Meijgaard E (2008) Recent Antarctic ice mass loss from radar interferometry and regional climate modelling. Nat Geosci $1: 106-110$

Rignot E, Velicogna I, van den Broeke MR, Monaghan A, Lenaerts J (2011) Acceleration of the contribution of the Greenland and Antarctic ice sheets to sea level rise. Geophys Res Lett 38(L05503):583. doi: 10.1029/2011GL046

Ritz C, Rommelaere V, Dumas C (2001) Modeling the evolution of the Antarctic ice sheet over the last 420000 years: implications for altitude changes in the Vostok region. J Geophys Res 106(D23): 31943-31964

Robison RAV, Huppert HE, Worster MG (2009) Dynamics of viscous grounding lines. J Fluid Mech 648:363-380. doi:10.1017/S0022112009993119

Schoof C (2007a) Ice sheet grounding line dynamics: steady states, stability and hysteresis. J Geophys Res 112(F03S28):664. doi:10.1029/2006JF000

Schoof C (2007b) Marine ice sheet dynamics. Part I. The case of rapid sliding. J Fluid Mech 573:27-55

Schoof C, Hindmarsh R (2010) Thin-film flows with wall slip: an asymptotic analysis of higher order glacier flow models. Quart J Mech Appl Math 63(1):73-114

Schoof C, Pattyn F, Hindmarsh R (2007) Benchmarks and intercomparison program for marine ice sheet models. Geophysical Research Abstracts 9(EGU2007-A-04644)

Shepherd A, Wingham DJ, Mansley JAD, Corr HFJ (2001) Inland thinning of Pine Island Glacier, West Antarctica. Science 291:862-864 
Thomas RH, Bentley CR (1978) A model for holocene retreat of the West Antarctic ice sheet. Quat Res 10:150-170

Velicogna I (2009) Increasing rates of ice mass loss from the Greenland and Antarctic ice sheets revealed by GRACE. Geophys Res Lett 36(L19503):222. doi:10.1029/2009GL040

Vieli A, Payne A (2005) Assessing the ability of numerical ice sheet models to simulate grounding line migration. J Geophys Res 110(F01003):202. doi:10.1029/2004JF000

Weertman J (1974) Stability of the junction of an ice sheet and an ice shelf. J Glaciol 13:3-11

Wingham DJ, Wallis DW, Shepherd A (2009) Spatial and temporal evolution of Pine Island Glacier thninning, 1995-2006. Geophys Res Lett 36(L17501):126. doi:10.1029/2009GL039 